\title{
Hepatocellular carcinoma: early-stage management challenges
}

This article was published in the following Dove Press journal:

Journal of Hepatocellular Carcinoma

23 June 2017

Number of times this article has been viewed

\author{
Derek J Erstad' \\ Kenneth K Tanabe ${ }^{2-4}$ \\ 'Department of Surgery, \\ Massachusetts General Hospital, \\ ${ }^{2}$ Harvard Medical School, ${ }^{3}$ Division \\ of Surgical Oncology, ${ }^{4}$ Massachusetts \\ General Hospital Cancer Center, \\ Massachusetts General Hospital, \\ Boston, MA, USA
}

\begin{abstract}
Hepatocellular carcinoma (HCC) is a major cause of cancer death and is increasing in incidence. This review focuses on HCC surveillance and treatment of early-stage disease, which are essential to improving outcomes. Multiple societies have published HCC surveillance guidelines, but screening efforts have been limited by noncompliance and overall lack of testing for patients with undiagnosed chronic liver disease. Treatment of early-stage HCC has become increasingly complex due to expanding therapeutic options and better outcomes with established treatments. Surgical indications for HCC have broadened with improved preoperative liver testing, neoadjuvant therapy, portal vein embolization, and perioperative care. Advances in post-procedural monitoring have improved efficacies of transarterial chemoembolization and radiofrequency ablation, and novel therapies involving delivery of radiochemicals are being studied in small trials. Finally, advances in liver transplantation have allowed for expanded indications beyond Milan criteria with non-inferior outcomes. More clinical trials evaluating new therapies and multimodal regimens are necessary to help clinicians design better treatment algorithms and improve outcomes.
\end{abstract}

Keywords: hepatocellular carcinoma, hepatitis, surveillance, Barcelona clinic liver cancer, staging, cirrhosis, hepatectomy, hepatic resection, locoregional therapy, radiofrequency ablation, transarterial chemoembolization, liver transplantation

\section{Introduction}

Hepatocellular carcinoma (HCC) accounts for $70 \%-90 \%$ of primary liver cancers and is the second leading cause of cancer death in East Asia and sub-Saharan Africa and the sixth most common in Western countries. ${ }^{1,2}$ The incidence of HCC in the US has tripled over the last 4 decades, which is attributable to multiple factors including undiagnosed chronic hepatitis $\mathrm{C}$ virus ( $\mathrm{HCV}$ ), alcohol consumption, and a rising incidence of obesity and metabolic syndromes. ${ }^{3,4} \mathrm{HCC}$ also represents the fastest rising cause of cancer-related death in the US and remains difficult to manage, with an average 5-year survival $<15 \%$ (Table 1 ). ${ }^{3}$ Contributing factors include limited responsiveness to systemic chemotherapy, association with chronic liver disease, and delayed diagnoses despite improved surveillance in developed regions. In this study, we describe the challenges and limitations associated with HCC surveillance and the impact these have on diagnosis and treatment. New technologies and expanded indications for standard therapies have led to improved patient outcomes. But choosing a therapeutic plan has become increasingly complex and requires consideration of multiple factors including tumor burden, baseline liver function, and comorbidities, usually by a multidisciplinary
Correspondence: Kenneth K Tanabe Division of Surgical Oncology, Massachusetts General Hospital, 55 Fruit Street, Boston, MA 02114-2696, USA

Tel +l 6177243868

Fax +l 6177243895

Email ktanabe@partners.org 
Table I HCC epidemiology

\section{HCC statistics}

Represents $70 \%-90 \%$ of all primary liver cancers ${ }^{\prime}$

Second most common cause of cancer-related mortality in East Asia and underdeveloped nations ${ }^{1,2}$

Sixth most common cause of cancer-related death in the US and Europe'

Fastest-rising cause of cancer-related death in the US $S^{3,4}$

Five-year survival remains $<15 \%^{3}$

Abbreviation: HCC, hepatocellular carcinoma.

team. In this context, we discuss the expanding role of ablative therapy, surgical resection, and transplantation for $\mathrm{HCC}$ in the modern era.

\section{HCC screening: a moving target}

HCC surveillance guidelines for at-risk populations have been put forth by multiple international organizations and vary in frequency and method of screening. ${ }^{5}$ Many clinicians in the United States follow the American Association of Liver Diseases (AASLD) consensus guidelines, which recommend surveillance for at-risk populations every 6 months with abdominal ultrasound (US). However, there remains uncertainty regarding optimal timing of screening, particularly for the highest risk patients. Two trials compared 6- and 12-month surveillance intervals, and no survival differences were found. ${ }^{6,7}$ However, a separate, large trial also comparing these time intervals did show several advantages with biannual screening, including smaller tumor size at diagnosis, a higher percentage of curative interventions, and improved survival. ${ }^{8}$ The 3 - and 4-month intervals have also been evaluated. More abnormal nodules were detected in the 3-month group compared to the 6-month group, although this led to more diagnostic investigations without evidence of increased HCC diagnosis or improved survival. ${ }^{9}$ Finally, 4- and 12-month intervals were compared in patients with chronic viral hepatitis. The 4-month interval detected more patients with early, resectable tumors, although this did not translate to a difference in 5-year overall survival. ${ }^{10}$ Unlike the AASLD, the Japanese Society of Hepatology (JSH) recommends screening every 3-4 months for the subset of higher risk patients who have concomitant viral hepatitis and cirrhosis. ${ }^{11}$ Notably, certain factors may abrogate the potential benefit of more frequent screening, including lack of follow-up testing for concerning lesions, length time bias, and difficultly differentiating early HCC from dysplasia. ${ }^{12}$ Nonetheless, there is low-strength evidence that surveillance improves outcomes at a population level. ${ }^{13}$
The use of alpha-fetoprotein (AFP) for HCC screening is also variably recommended among international organizations. AFP is no longer recommended by Western societies due to its low positive predictive value in these populations, which have a lower HCC prevalence compared to East Asian populations. Lok et al evaluated AFP for its potential in diagnosing HCC prior to clinical detection in the Hepatitis $\mathrm{C}$ Antiviral Longterm Treatment against Cirrhosis (HALT-C) trial population, which includes patients with chronic $\mathrm{HCV}$ and documented bridging fibrosis on biopsy. Among those with eventual HCC, US detected a suspicious nodule prior to diagnosis in $58 \%$ of cases, whereas AFP was elevated in only $20 \%$ of patients. AFP elevation is more apparent in established disease but appears to provide little value for preclinical diagnosis. The authors also trialed a lower serum AFP threshold to obtain a higher sensitivity of at least $90 \%$, though this was associated with a significant drop in AFP specificity to 15\%-57\%. ${ }^{14}$ Higher baseline serum AFP levels in cirrhotic patients, presumably due to chronic hepatocyte injury, further compound this lack of specificity. ${ }^{15}$ More recently, several meta-analyses have investigated the value of AFP and other serum biomarkers in screening and have similarly shown variable sensitivity and specificity. ${ }^{16}$

AFP screening has also demonstrated mixed cost-effectiveness in at-risk populations due to differences in disease prevalence and available resources. In resource-limited settings, AFP does appear to provide cost-savings value. ${ }^{17}$ However, in non-resource-limited settings, the value of AFP in addition to US compared to US alone has only been evaluated in several studies. Saab et $\mathrm{al}^{18}$ compared US, US plus AFP, and CT in a hypothetical cohort awaiting liver transplantation for end-stage liver disease using a Markov model and found that the incremental cost-effectiveness ratio was $\$ 60.30$ per life-year for US alone compared to $\$ 74.00$ for US plus AFP. Based on published analyses, AFP does not appear to represent a large economic burden, though in settings where it has been shown to provide little or no additional value, the cost is not justified. A summary of commonly used screening guidelines published by different national liver societies is shown in Table 2.

\section{Surveillance noncompliance among at-risk populations}

Despite rigorous evaluation of optimal screening frequency, surveillance noncompliance in the US and abroad remains an important contributing factor to delayed diagnosis of HCC. Among patients with known risk factors in the US, less 
Table 2 Comparison of commonly used HCC screening guidelines

\begin{tabular}{|c|c|c|c|c|c|}
\hline & AASLD $^{98}$ & $\mathbf{N C C N}^{38}$ & EASL-EORTC $^{99}$ & JHS $^{100}$ & APASL ${ }^{101}$ \\
\hline $\begin{array}{l}\text { Population } \\
\text { recommended to } \\
\text { undergo screening }\end{array}$ & $\begin{array}{l}\text { Cirrhosis of any } \\
\text { etiology }\end{array}$ & $\begin{array}{l}\text { Cirrhosis of any } \\
\text { etiology } \\
\text { HBV carriers with } \\
\text { additional risk } \\
\text { factors* }\end{array}$ & $\begin{array}{l}\text { Cirrhosis of any etiology } \\
\text { HBV carriers with active } \\
\text { infection or family history } \\
\text { of HCC } \\
\text { HCV carriers with } \\
\text { advanced fibrosis }\end{array}$ & $\begin{array}{l}\text { Extremely high-risk: cirrhosis } \\
\text { due to HBV or HCV } \\
\text { High-risk: HBV or HCV, } \\
\text { cirrhosis of any other etiology }\end{array}$ & $\begin{array}{l}\text { Cirrhosis due to } \\
\mathrm{HBV} \text { or } \mathrm{HCV}\end{array}$ \\
\hline $\begin{array}{l}\text { Abdominal US } \\
\text { screening frequency }\end{array}$ & 6 months & $6-12$ months & 6 months & $\begin{array}{l}\text { Extremely high-risk: } 3-4 \text { months } \\
\text { High-risk: } 6 \text { months }\end{array}$ & 6 months \\
\hline Serum markers & Not recommended & Not recommended & Not recommended & $\begin{array}{l}\text { Recommend: AFP, DCP, and } \\
\text { AFP-L3\% } \\
\text { Extremely high-risk group: } \\
\text { every 3-4 months } \\
\text { High-risk group: every } 6 \text { months }\end{array}$ & Recommend AFP \\
\hline $\begin{array}{l}\text { Axial imaging for } \\
\text { concerning nodules }\end{array}$ & $\begin{array}{l}\text { Four-phase } \\
\text { contrast-enhanced } \\
\text { CT } \\
\text { Gadolinium- } \\
\text { enhanced MRI }\end{array}$ & $\begin{array}{l}\text { Four-phase contrast- } \\
\text { enhanced CT } \\
\text { Gadolinium-enhanced } \\
\text { MRI }\end{array}$ & $\begin{array}{l}\text { Four-phase contrast- } \\
\text { enhanced CT } \\
\text { Gadolinium-enhanced } \\
\text { MRI }\end{array}$ & $\begin{array}{l}\text { Four-phase contrast-enhanced } \\
\text { CT } \\
\text { Gd-EOB-DTP-enhanced MRI }\end{array}$ & $\begin{array}{l}\text { Four-phase contrast- } \\
\text { enhanced CT } \\
\text { Gadolinium-enhanced } \\
\text { or SPIO MRI }\end{array}$ \\
\hline CEUS & Not recommended & Not recommended & Not recommended & Sonazoid contrast & $\begin{array}{l}\text { Sonazoid or Levovist } \\
\text { contrast }\end{array}$ \\
\hline $\begin{array}{l}\text { Accepted methods } \\
\text { for diagnosis }\end{array}$ & $\begin{array}{l}\text { Axial imaging } \\
\text { Core biopsy }\end{array}$ & $\begin{array}{l}\text { Axial imaging } \\
\text { Core biopsy }\end{array}$ & $\begin{array}{l}\text { Axial imaging } \\
\text { Core biopsy }\end{array}$ & $\begin{array}{l}\text { Axial imaging } \\
\text { CEUS } \\
\text { Core biopsy }\end{array}$ & $\begin{array}{l}\text { Axial imaging } \\
\text { CEUS } \\
\text { Core biopsy }\end{array}$ \\
\hline
\end{tabular}

Notes: *Additional risk factors include HBV carrier with family history of HCC, Asian males $\geq 40$ years, Asian females $\geq 50$ years, and African/North American Blacks with HBV.

Abbreviations: AASLD, American Association for the Study of Liver Diseases; AFP, alpha-fetoprotein; APASL, Asian Pacific Association for the Study of the Liver; CEUS, contrast-enhanced ultrasound; CT, computed tomography; DCP, des-gamma-carboxy prothrombin; EASL-EORTC, European Association for the Study of the Liver European Organisation for Research and Treatment of Cancer; Gd-EOB-DTP, gadolinium-ethoxybenzyl-diethylentriamine pentaacetic acid; HBV, hepatitis B virus; HCC, hepatocellular carcinoma; HCV, hepatitis C virus; JHS, Japan Society of Hepatology; MRI, magnetic resonance imaging; NCCN, National Comprehensive Cancer Network; SPIO, superparamagnetic iron oxide; US, ultrasound.

than one-third are up to date with recommended screening guidelines. ${ }^{19-21}$ Providers, patients, and the American health care system all contribute to failed surveillance programs. ${ }^{22}$ Among health care providers, patients followed in gastroenterology specialty clinics are more likely to be screened according to guidelines. ${ }^{23}$ This finding was corroborated by a review of patients coinfected with HIV and hepatitis B virus (HBV) who were followed primarily in infectious disease clinics. These individuals underwent significantly fewer recommended screenings compared to patients monoinfected with HBV who were regularly seen by a hepatologist. ${ }^{24}$ Similarly, patients followed in transplant clinics were also found to have overall better compliance with recommended surveillance guidelines. ${ }^{25}$ Certain patient factors have been associated with screening adherence. Henrion et $\mathrm{al}^{26}$ showed that etiology of liver disease, particularly alcohol-related cirrhosis, was associated with worse surveillance compliance, especially for patients with continued use. Other factors including male sex and patient involvement in care have been associated with improved compliance on multivariate analysis, whereas non-Caucasian race and low socioeconomic status have been associated with less surveillance. ${ }^{23,27}$ Regarding the health care system, cost of testing, difficulty scheduling, and limited transportation options have been reported as barriers to regular screening. ${ }^{28}$ However, insurance status does not appear to improve compliance. ${ }^{19}$ In summary, screening failure likely results from a complex interplay of multiple deterring factors, and more trials focused on surveillance adherence are necessary to ultimately increase the percentage of early-stage diagnoses.

\section{Unknown chronic liver disease: a silent killer}

In addition to poor surveillance compliance among known atrisk populations, a second major contributing factor to delayed diagnosis of HCC is that many patients with chronic liver disease are unaware of their condition and therefore receive no care specific for it. In the USA, this is mainly driven by HCV infection. Approximately $1.6 \%$ of the population is infected with $\mathrm{HCV}$, though three-quarters of patients are unaware of their infection. Globally, $<5 \%$ of $\mathrm{HCV}$-positive patients are aware of their infection status (Figure 1A). ${ }^{29}$ Nearly $80 \%$ of 
A

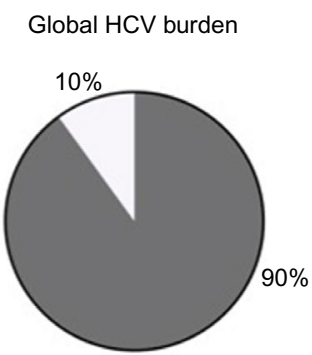

Estimated number of infections (>184 million) $\square$ Proportion aware of infection status (<10\%)

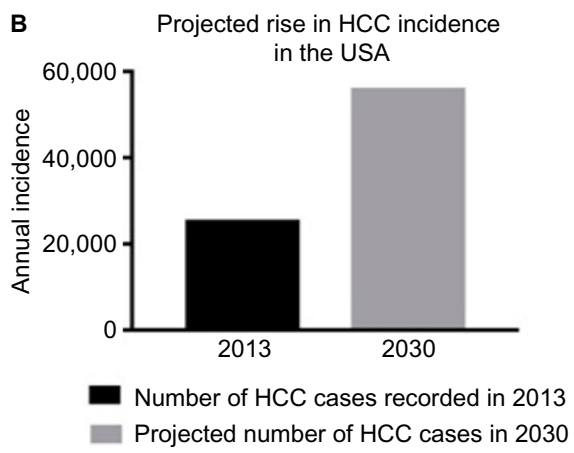

Figure I HCV global burden and rising incidence of $\mathrm{HCC}$ in the USA.

Notes: (A) HCV global statistics from adapted by permission from Macmillan Publishers Ltd: Nat Rev Gastroenterol Hepatol, Thrift AP, El-Serag HB, Kanwal F, Global epidemiology and burden of Hcv infection and Hcv-related disease, 2017;14(2):122-132, copyright 2016. ${ }^{107}$ (B) Projected HCC incidence data reprinted with permission (C2016 American Society of Clinical Oncology. All rights reserved. Petrick JL, Kelly SP, Altekruse SF, McGlynn KA, Rosenberg PS. Future of hepatocellular carcinoma incidence in the United States forecast through 2030. J Clin Oncol. 2016;34(I5): I787-1794. ${ }^{108}$

Abbreviations: $\mathrm{HCC}$, hepatocellular carcinoma; $\mathrm{HCV}$, hepatitis $\mathrm{C}$ virus.

HCV patients develop chronic hepatitis, from which $15 \%$ will progress to cirrhosis. The risk of $\mathrm{HCC}$ among cirrhotic patients is $\sim 3 \%$ per year. The progression from infection to cirrhosis and HCC occurs over a few decades, and most patients diagnosed with $\mathrm{HCC}$ are $>60$ years old. Although the annual number of total infections is declining, the prevalence of the diagnoses of HCV cirrhosis and HCC in the US continues to rise because the majority of patients were infected before knowledge of the virus and screening technology (Figure 1B). ${ }^{30}$ Most HCV-infected patients will not experience symptoms until they have developed advanced liver disease. The impact of effective $\mathrm{HCV}$ antiviral therapies on $\mathrm{HCC}$ incidence is not well established, although it is known that patients with cirrhosis are still at risk for $\mathrm{HCC}$ after obtaining a sustained virologic response (SVR). ${ }^{31}$ Therefore, the Centers for Disease Control (CDC) proposed a series of HCV screening recommendations based on risk exposures. ${ }^{32}$ However, risk-based screening guidelines did not gain traction among the medical community, and therefore, in 2012, the CDC presented new recommendations based upon birth year. The CDC is primarily focused on baby boomers born between 1945 and 1965, as this subset of the population has the highest HCV prevalence, which is nearly $3 \%{ }^{33}$ The updated guidelines recommend one-time HCV testing for all persons born during 1945-1965, regardless of HCV risk. Long-term studies are needed to determine the effectiveness of this approach.

As a consequence of undiagnosed chronic liver disease and poor surveillance compliance, only $3 \%$ of newly diagnosed patients meet criteria for very early disease and $25 \%$ for early disease, as defined by the Barcelona Clinic Liver Cancer (BCLC) staging classification. ${ }^{34,35}$ Very early HCC (Stage 0 ) is limited to a single tumor $<2 \mathrm{~cm}$ in diameter in a patient with asymptomatic Child-Pugh A class liver disease. Early HCC (Stage A) is limited to a single tumor $<5 \mathrm{~cm}$ in diameter or three tumors that are each $<3 \mathrm{~cm}$ in diameter. In addition, patients must have either Child-Pugh A or B liver disease without symptoms or change in performance status. ${ }^{36}$ Although there are conflicting reports, it has been shown that very early-stage disease patients survive longer than those with early stage.$^{34}$ In combination, these groups make up the $10 \%-30 \%$ of HCCs amenable to surgical resection, with the remainder of patients relegated to transplant waiting lists, palliative therapy, and higher mortality. ${ }^{34}$

\section{Diagnostic uncertainty: navigating cirrhosis, dysplasia, and early HCC}

Cirrhotic nodules, dysplasia, and early HCC are often difficult to differentiate on imaging and histopathology, adding complexity to the diagnosis of very early- and early-stage disease. The AASLD recommends US for initial HCC screening, followed by axial imaging for suspicious nodules. Contrastenhanced ultrasound (CEUS) and AFP are not recommended by the AASLD or National Comprehensive Cancer Network (NCCN). ${ }^{36-38}$ The diagnostic sensitivity of US decreases in proportion to tumor size, nearing $63 \%$ for early-stage lesions. ${ }^{39}$ To prevent overdiagnosis of noncancerous lesions, nodules $<1 \mathrm{~cm}$, which are unlikely to be HCC, are monitored with increased surveillance every 3 months. ${ }^{36} \mathrm{HCC}$ tumor doubling time ranges from 1 to 12 months; therefore, this interval is thought to be sufficient to appropriately diagnose the majority of fast-growing $\mathrm{HCC}$ before disease progression. ${ }^{40} \mathrm{In}$ contrast, lesions $>1 \mathrm{~cm}$ are more likely to be HCC and are subjected to axial imaging with either triple-phase computed tomography (CT) or contrast-enhanced magnetic resonance imaging (MRI). ${ }^{41}$ Mature HCCs display arterial hypervascular enhancement with washout on the venous phase, a radiographic quality that is distinct from the surrounding hepatic parenchyma. This pattern of contrast phasing is highly specific 
for HCC and is sufficient to establish a diagnosis without biopsy. ${ }^{36}$ However, early HCCs are well differentiated and less radiographically distinct in $>50 \%$ of cases, thus requiring a biopsy for diagnosis. ${ }^{42}$ Percutaneous biopsy in a cirrhotic liver is prone to erroneous sampling, and early HCCs can be difficult to distinguish on histology from surrounding cirrhotic tissue and dysplastic nodules. ${ }^{43}$ Therefore, patients with suspicious imaging followed by a negative or indeterminate biopsy are subjected to increased surveillance with abdominal US every 3 months to assess for progression of suspicious nodules. ${ }^{36}$ Unfortunately, there are still no available serum markers with sufficient sensitivity and specificity to aid in diagnosis during such instances in which both imaging and tissue sampling fail to yield a definitive result.

\section{Navigating treatment options for early-stage disease}

Multiple treatment modalities are available for HCC including local ablative therapy, embolization, surgical resection, liver transplantation, and systemic chemotherapy. The decision analysis for treatment should incorporate patient comorbidities and offer the greatest opportunity for complete tumor eradication while considering procedural risks. The high prevalence of advanced liver disease in the HCC population precludes many patients from curative resection. Establishing an individualized treatment plan is best done with a multidisciplinary team of surgeons, oncologists, and interventional radiologists, generally at a tertiary care center that is well trained in managing chronic liver disease. ${ }^{44}$

\section{Expanding indications for surgical resection of HCC}

The decision to pursue surgical resection for HCC is determined by the size and location of the tumor, the patient's baseline liver function, and comorbid conditions. ${ }^{45}$ Due to the high frequency of underlying liver disease associated with HCC, liver surgeons are forced to balance competing objectives, which are to provide an oncologic resection with adequate negative margins while minimizing the volume of hepatic parenchyma resected. In support of these principles, current AASLD guidelines recommend limiting resection to patients with very early- and early-stage HCC. ${ }^{46}$ To be considered for surgery, cirrhotic patients generally must have preserved hepatic function without evidence of clinically significant portal hypertension or hyperbilirubinemia and their disease burden is ideally limited to a unifocal lesion without macrovascular invasion. ${ }^{47}$ When reviewing outcomes among all resectable patients, those with solitary $\mathrm{HCC}<5 \mathrm{~cm}$ in diameter in the absence of cirrhosis have the greatest longterm survival. ${ }^{48}$ By current guidelines, patients with multiple HCC, very large tumors, or macrovascular invasion are often not considered operative candidates.

However, improvements in liver surgery technique and perioperative care have led to expanded criteria for HCC resection in some tertiary centers. The East-West Study Group recently completed a multinational retrospective analysis in which 2,046 patients with HCC staged beyond Milan criteria who were treated with hepatic resection and evaluated for survival outcomes. Fifty percent of patients were categorized as BCLC stage B or higher; yet, all stages had similar 5-year disease-specific survival. The reported 3- and 5-year overall survival for BCLC stages B and C patients was $62 \%$ and $50 \%$, respectively, markedly greater than the expected survival with treatment based on consensus guidelines. Interestingly, multifocal HCC was not considered a risk factor for poor outcome on multivariate analysis, though tumor size and macrovascular invasion were. ${ }^{49}$ Similar survival outcomes were reported in two separate, large analyses of patients with HCC beyond Milan criteria who were treated with resection. ${ }^{50,51}$ These findings suggest that surgery for expanded indications with respect to tumor size and multiplicity is a reasonable treatment option for a subset of patients.

\section{Navigating cirrhosis in operative planning}

Multiple techniques have been developed to facilitate hepatic resection in the setting of cirrhosis. This is particularly relevant for patients with solitary, early-stage HCC who could otherwise be cured with hepatectomy but have limiting chronic liver disease. Postoperative liver failure is the major source of morbidity and mortality after resection, and preoperative physiologic and biochemical markers of liver function do not provide a reliable prediction of postoperative outcomes. ${ }^{52}$ One approach to address this problem has been to develop techniques to characterize the future liver remnant (FLR), which represents the remaining liver after resection. FLR calculations are rooted in either volumetric or functional algorithms, though the two approaches do not appear to be equally reliable. Although liver size and function correlate under normal conditions, this relationship devolves with cirrhosis, and therefore, functional tests have been shown to be more accurate predictors of operative outcome. ${ }^{53}$ Nonetheless, a volumetric FLR $>20 \%$ in patients with healthy livers and $>40 \%$ in the setting of chronic liver disease are established standards. ${ }^{54}$ In multiple reviews, both 
volumetric and functional FLR have been shown to reduce postoperative liver failure and mortality in cirrhotic patients undergoing major hepatectomy. ${ }^{55}$

For cirrhotic patients with insufficient predicted FLR, portal vein embolization (PVE) has been shown to significantly reduce the incidence of postoperative complications related to liver failure. ${ }^{56} \mathrm{PVE}$ is a preoperative technique that induces hyperplasia of contralateral hepatic parenchyma, increasing the size and function of the FLR, and thereby reducing the risk of postoperative liver failure. The mechanism of action underlying PVE is multifactorial. First, reduced blood flow to hepatocytes results in the release of growth factors that stimulate cellular division and proliferation in the remaining healthy parenchyma via paracrine signaling. Second, augmentation in portal venous pressure and flow is also thought to stimulate hepatic growth. This logic is predicated on evidence that the greatest amount of regeneration is seen in the periportal region, though finer details regarding the exact mechanism are not well established. ${ }^{57}$ The goal of PVE is to enable surgical resection that could not be performed safely without growth of the predicted FLR.

When compared with transarterial chemoembolization (TACE), PVE followed by hepatectomy has been shown to provide superior 5-year survival outcomes. ${ }^{58}$ More recently, combined preoperative PVE and TACE followed by hepatectomy has also been investigated as a means to both downstage advanced disease for resection and reduce the risk of postoperative liver failure..$^{59}$

Finally, there is ongoing debate regarding the impact of clinically significant portal hypertension on hepatectomy outcomes. Historically, the presence of portal hypertension has been considered an absolute contraindication to surgery in HCC. However, multiple retrospective analyses have shown that portal hypertension is less important than baseline model end-stage liver disease (MELD) and extent of hepatectomy, and some authors recommended that it no longer be considered an absolute contraindication to resection. ${ }^{60,61}$ However, a meta analysis of 11 studies that specifically addressed portal hypertension in hepatectomy patients concluded that it was an independent predictor of mortality at 3 and 5 years, though progression-free survival and disease-free survival were not evaluated in this study. ${ }^{62}$ Uncertainty on the topic remains for multiple reasons, including a paucity of prospective data and limited subgroup analyses in retrospective studies. Specifically, portal hypertension may have variable significance depending on the degree of hypertension, the severity of associated cirrhosis, the tumor burden and presence or absence of vascular invasion, and the planned size of hepatic resection. Surgeons are thus left to consider the significance of portal hypertension on an individual basis until updated consensus guidelines are established.

\section{Laparoscopic versus open hepatectomy}

Laparoscopic liver resection was first pioneered in the 1990s, though it was initially slow to gain popularity. Concerns among surgeons included loss of tactile feedback in an already challenging operation, reduced ability to control bleeding, injury to the biliary system, and gas embolism. However, refinements in technique and improved technologies, such as energy sources for coagulation of liver parenchyma, led to better outcomes and increased adoption by hepatobiliary surgeons. Multiple institutions have published their experience with laparoscopic hepatectomy compared to open surgery, with overall promising results. ${ }^{63}$ Importantly, there does not appear to be a difference in oncologic outcomes. Cheung et al performed a long-term survival analysis of pure laparoscopic versus open hepatectomy for $\mathrm{HCC}$ and found no difference in survival outcomes between techniques, albeit a small sample size (32 laparoscopic cases versus 64 case match control open resection cases). Laparoscopy was associated with less blood loss, shorter hospital stay, and fewer postoperative complications. ${ }^{64}$ Multiple other groups have reported similar improvements in hospital length of stay and reduced complications. ${ }^{65-67}$

Laparoscopy is most commonly confined to minor hepatectomy and is ideally suited for superficial lesions, though many centers now routinely perform laparoscopic hemihepatectomies. For patients with cirrhosis, this approach may be better tolerated than open surgery and may allow for expanded indications for surgical resection. ${ }^{68}$ It is not established yet what the limits of laparoscopic hepatectomy will be, if any, in terms of size and location of resection. ${ }^{69}$ Nonetheless, it is likely that laparoscopy will continue to have an increasing role in the operative management of HCC, particularly as current trainees are increasingly exposed to this technique.

\section{Radiofrequency ablation versus hepatectomy for early-stage HCC}

The choice between Radiofrequency ablation (RFA) and liver resection remains a controversial topic. Historically, most studies have shown that surgery provides a higher rate of cure, longer disease-free survival, and improved overall survival. ${ }^{70}$ There have been three randomized controlled 
trials designed to compare RFA and surgical resection for early-stage HCC. The only trial showing a significant difference was performed by Huang et al, in which 230 patients with BCLC stage A HCC were randomized to receive RFA or surgical resection. Surgical resection provided significantly longer overall survival and recurrencefree survival and lower recurrence rate at 5 years. ${ }^{71}$ In a separate trial by Lu et al, 105 patients with BCLC stage A HCC were randomized to receive percutaneous RFA or surgical resection. There was no difference in overall survival or treatment-related complication, though there was a nonsignificant trend toward improved disease-free survival with surgical resection. ${ }^{72}$ Finally, Feng et al randomized 168 patients with $\mathrm{HCC}<4 \mathrm{~cm}$ in diameter and up to two nodules to RFA or surgical resection. There was no difference in overall survival or recurrence-free survival at 3 years. However, there was a trend toward higher recurrence in the RFA group. ${ }^{73}$

Although most current evidence supports the use of surgical resection over RFA, for appropriate lesions $<3.5 \mathrm{~cm}$, RFA offers the advantage of a less invasive intervention, shorter hospital stay, and faster recovery. And it is particularly useful for patients with advanced liver disease who cannot tolerate hepatectomy. Moreover, advances in RFA technique have led to improved outcomes. One such approach involves cotreatment with RFA and an additional modality, such as radiation seeds or TACE. Clinical trials evaluating multimodal therapies are shown in Table 3.

Improvements in post-procedure monitoring for tumor progression and recurrence have also allowed for better detection of small nodules and recurrent HCC after locoregional therapy. ${ }^{74,75}$ Hepatobiliary phase MRI with hepatocyte-specific contrast agents including gadoxetic acid and gadobenate dimeglumine has been shown in multiple trials to provide better diagnostic sensitivity for HCC compared to the current standard multidetector CT (MDCT) ${ }^{76} \mathrm{Kim}$ et $\mathrm{al}^{75}$ compared imaging with gadoxetic $\mathrm{MR}$ and $\mathrm{CT}$ versus $\mathrm{CT}$ alone and found that MR upstaged $13 \%$ of patients with $\mathrm{HCC}$ by BCLC criteria, which changed their management plans and led to improved long-term survival. Gadoxetic-based MRI has also been applied in the acute post-RFA period as a means to grade the ablative margin radiographically. In a trial of 124 HCC patients, Koda et al ${ }^{77}$ were able to use this approach to accurately stratify patients who underwent RFA based on their risk for local recurrence.

Given its advantages, hepatocyte-specific contrast imaging could improve HCC care in several ways. First, it may provide more clarity regarding diagnostic uncertainty of small nodules in cirrhotic livers. Second, it may allow for more accurate post-procedural monitoring after locoregional therapy or surgery. In this regard, a recent, large trial comparing RFA and surgical resection for patients with very earlystage HCC found that surgery provided superior outcomes with respect to both recurrence and survival. However, on subgroup analysis, among patients that recurred, there was no difference in survival between treatment groups. ${ }^{78}$ This suggests that increased recurrence is the driver of worse prognosis after ablative therapy, and therefore, a method detecting earlier recurrence may ameliorate differences in outcomes between locoregional treatment and surgery. Third, given the

Table 3 Clinical trials evaluating multimodal locoregional therapies

\begin{tabular}{|c|c|c|c|c|}
\hline Author & Year & Enrollment and inclusion criteria & Treatment & Findings \\
\hline Azab et $\mathrm{al}^{102}$ & 2011 & $\begin{array}{l}90 \text { patients } \\
\mathrm{BCLC} \text { stage 0-A HCC }\end{array}$ & $\begin{array}{l}\text { RFA + percutaneous } \\
\text { ethanol injection versus } \\
\text { RFA monotherapy }\end{array}$ & $\begin{array}{l}\text { RFA + PEI associated with improved survival at } \\
18 \text { months for lesions sized } 3.1-5.0 \mathrm{~cm}(p=0.03)\end{array}$ \\
\hline Peng et $\mathrm{a}^{103}$ & 2013 & $\begin{array}{l}\text { I89 patients } \\
\text { Single } \mathrm{HCC}<7 \mathrm{~cm} \text { or three lesions each }<3 \mathrm{~cm} \\
\text { Child-Turcotte-Pugh A or B cirrhosis }\end{array}$ & $\begin{array}{l}\text { RFA + TACE versus } \\
\text { RFA monotherapy }\end{array}$ & $\begin{array}{l}\text { RFA }+ \text { TACE associated with improved overall } \\
\text { survival }(p=0.002) \text { and recurrence-free survival } \\
(p=0.009) \text { at } 3 \text { years }\end{array}$ \\
\hline Bian et $\mathrm{al}^{104}$ & 2014 & $\begin{array}{l}\text { I27 patients } \\
\text { BCLC stage 0-B HCC }\end{array}$ & $\begin{array}{l}\text { RFA + radioconjugated } \\
{\left[1^{|3|}\right] \text { metuximab versus }} \\
\text { RFA monotherapy }\end{array}$ & $\begin{array}{l}\text { Radiolabeled metuximab co-therapy with RFA } \\
\text { associated with longer recurrence-free survival } \\
(p=0.03)\end{array}$ \\
\hline Chen et al ${ }^{105}$ & 2014 & $\begin{array}{l}167 \text { patients } \\
B C L C \text { stage } 0-A H C C\end{array}$ & $\begin{array}{l}\text { RFA + iodine }{ }^{125} \\
\text { radiation seeds versus } \\
\text { RFA monotherapy }\end{array}$ & $\begin{array}{l}\text { RFA + iodine } e^{125} \text { associated with reduced } \\
\text { recurrence at } 5 \text { years }(p=0.004) \text { and improved } \\
5 \text {-year overall survival }(p=0.003)\end{array}$ \\
\hline Liu et $\mathrm{al}^{106}$ & 2016 & $\begin{array}{l}200 \text { patients } \\
\text { BCLC Stage 0-A HCC }\end{array}$ & $\begin{array}{l}\text { RFA + TACE versus } \\
\text { hepatectomy }\end{array}$ & $\begin{array}{l}\text { Hepatectomy associated with improved } 5 \text {-year } \\
\text { overall survival }(p=0.007) \text { and recurrence-free } \\
\text { survival }(p=0.026) \text {, though surgery associated with } \\
\text { higher complication rate }(p=0.026)\end{array}$ \\
\hline
\end{tabular}

Abbreviations: BCLC, Barcelona Clinic Liver Cancer; HCC, hepatocellular carcinoma; PEI, percutaneous ethanol injection; PVE, portal vein embolization; RFA, radiofrequency ablation; TACE, transarterial chemoembolization. 
high rate of HCC recurrence due to baseline liver disease in a majority of patients, chemopreventative strategies are an area of active investigation, which could have a beneficial impact on this patient population. Hepato-pancreato-biliaryspecific MR may provide a method for stratifying patients based on recurrence risk to determine who should be treated with chemopreventative therapy.

Thus, advances in both ablative therapy and liver-specific imaging are likely to improve outcomes with locoregional therapy, and the decision to pursue RFA versus surgery will become increasingly complex, particularly for patients who can withstand surgical resection. Considering the current capabilities of locoregional therapy and surgical resection, we propose the following treatment algorithm for the management of early-stage HCC (Figure 2). This updated approach incorporates combinatorial therapy, specifically neoadjuvant locoregional therapy for borderline resectable disease, as well as expanded indications for surgical resection. Going forward, clinical trials designed to investigate these novel technologies in comparison to standard therapies will be critical to provide objective information for clinicians.

\section{Advances in liver transplantation for HCC}

Liver transplantation offers the potential of cure for patients with early-stage HCC in the setting of end-stage liver disease and for patients with unresectable HCC. Advantages to transplant include complete extirpation of cancerous tissue and at-risk cirrhotic liver, reduced risk for local recurrence, and reversal of liver failure. However, for resectable patients with preserved liver function, there remains controversy regarding benefits of transplantation versus surgery. Multiple tertiary centers have

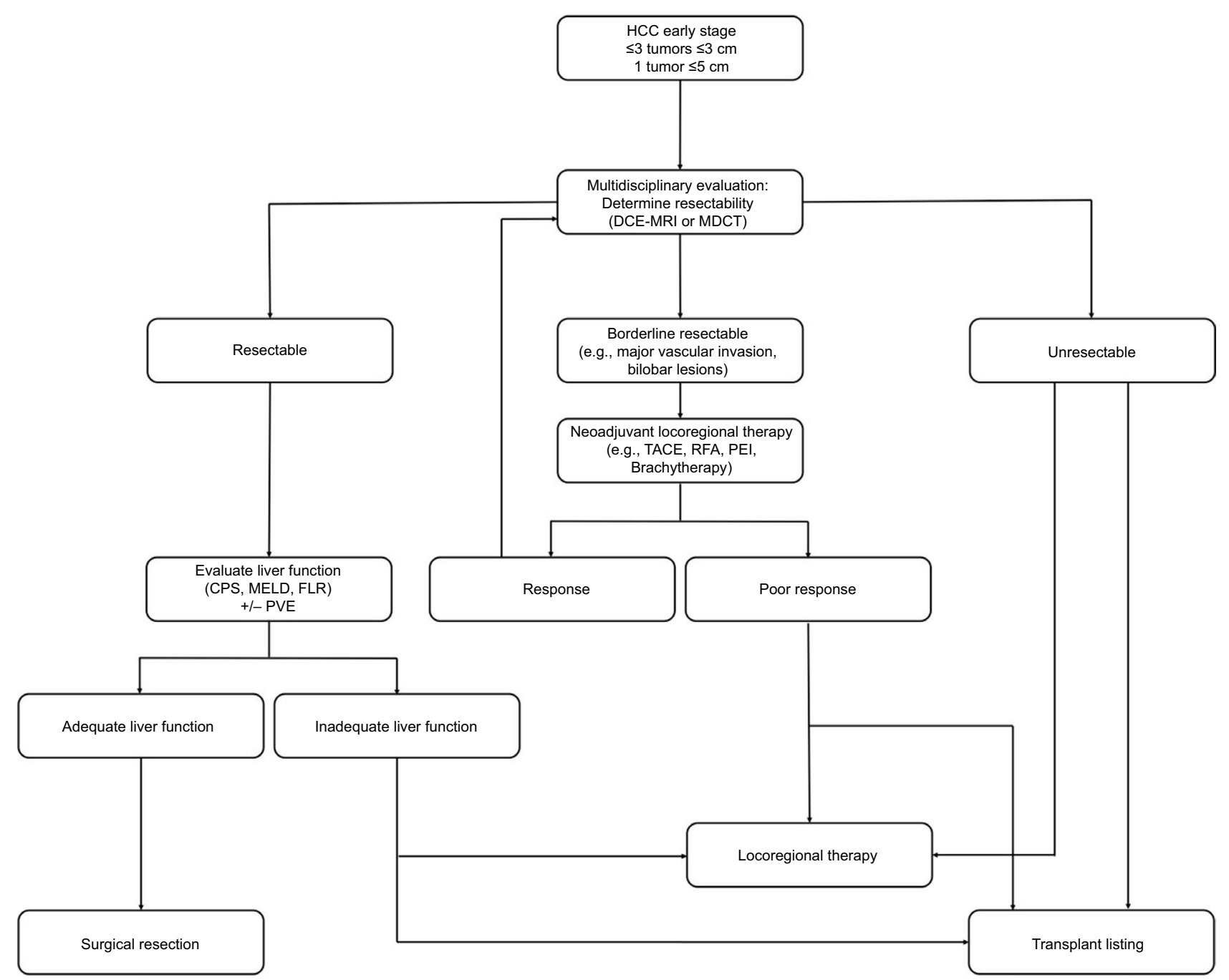

Figure 2 Early-stage HCC treatment algorithm.

Abbreviations: CPS, Child-Pugh score; DCE-MRI, dynamic contrast-enhanced magnetic resonance imaging; FLR, future liver remnant; HCC, hepatocellular carcinoma; MDCT, multidetector computed tomography; MELD, model end-stage liver disease; PEI, percutaneous ethanol injection; PVE, portal vein embolization; RFA, radiofrequency ablation; TACE, transarterial chemoembolization. 
compared their experience with both treatments, with most finding no difference in survival measures. ${ }^{79}$ Recently, Koniaris et al published the largest review to date of patients treated with either resection or transplantation. Interestingly, resection provided superior overall survival among patients with preserved liver function $(\mathrm{MELD}<10)$ who met either Milan or University of California San Francisco (UCSF) criteria when compared with transplantation. Two factors appeared to adversely impact transplant results. First, transplant is associated with a higher mortality within the first 2 years after procedure, likely related to graft dysfunction or immunosuppressive complications. Second, a portion of patients progressed while waiting for transplant. On subgroup analysis, comparing only those who went on to transplant versus surgery, there was no difference in 5 -year survival. However, transplantation was associated with longer 5-year recurrence-free survival compared to resection in patients with advanced MELD. ${ }^{80}$

Thus, as revealed by these findings, HCC progression while waiting for transplant remains a critical problem. Demand for organs has outpaced supply in recent years. ${ }^{81}$ The percentage of transplants for $\mathrm{HCC}$ has markedly increased to $>20 \%$ in the last decade. ${ }^{82}$ Multiple attempts have been made to modulate the fraction of grafts allocated to $\mathrm{HCC}$ patients, one of which is the adoption of exception points by the United Network for Organ Sharing (UNOS). Priority scoring is calculated based on risk assessment of HCC mortality, with a goal of achieving transplant within 6-12 months in most regions. ${ }^{83}$ Although this intervention has reduced wait-list time and death from disease, the 1-year dropout rate from disease progression remains between $15 \%$ and $30 \% .{ }^{84}$

Another approach to improve HCC outcomes through transplantation has been to expand transplant indications beyond the Milan criteria. Initial forays into liver transplantation in the 1960 s to 1980 s were plagued by frequent selection of patients with advanced $\mathrm{HCC}$, high recurrence rates, and poor outcomes. ${ }^{85}$ In 1996, UNOS adopted the Milan criteria, thereby providing an upper limit of acceptable tumor burden that standardized transplantation for HCC across the United States and markedly improved outcomes. ${ }^{86}$ However, with improvements in bridging therapy, tumor staging via cross-sectional imaging, and perioperative care, it has been shown that good outcomes are obtainable for patients with HCC beyond these criteria. ${ }^{87,88}$ The strongest evidence for this argument is seen in studies of results using the UCSF criteria, which allows for a larger tumor burden. In a review of the University of California Los Angeles liver transplant experience, Duffy et al evaluated 467 patients who underwent transplant for HCC and were stratified by stage, which included the Milan criteria, UCSF criteria, and a third group for patients beyond UCSF criteria. The authors found no significant difference in 5-year post-transplant survival between Milan and UCSF groups, though patients beyond UCSF criteria did markedly worse. On multivariate analysis, tumor number, lymphovascular invasion, and poor differentiation were associated with worse outcome. ${ }^{89}$ In a separate review, Mazzaferro et al analyzed 1,556 patients who underwent transplant for HCC, of which 1,112 patients were staged beyond the Milan criteria. This latter group did worse overall, though subgroup analysis revealed that patients beyond Milan criteria without microvascular invasion who still met the up-to-seven rule (seven as the sum of the largest tumor in centimeters plus number of tumors) did nearly as well as all patients meeting Milan criteria. ${ }^{88}$ Although this study used different criteria, it provides additional evidence that transplantation can provide good outcomes for appropriately selected patients under expanded criteria.

Tumor downstaging with neoadjuvant locoregional therapy and chemoembolization has also been used to broaden transplant eligibility. UNOS currently grants exception points only to patients with tumor-node-metastasis (TNM) stage II HCC, and those with more advanced disease are unlikely to obtain a sufficient MELD based on liver dysfunction. However, a patient may qualify for priority scoring through adequate downstaging. In this regard, Chapman et al evaluated 76 patients with $\mathrm{HCC}$ beyond Milan criteria who were treated with TACE. Eighteen patients (23\%) were sufficiently downstaged to undergo orthotopic liver transplant, and their survival outcomes were expectedly better compared to those who did not qualify. At the end of the trial, only one patient had recurrence, and there were no disease-related mortalities. ${ }^{90}$ Interestingly, the liver explants of downstaged patients showed upwards of $90 \%$ tumor necrosis in $75 \%$ of patients, which is higher than average for TACE therapy. It has been shown that adequate tumor downstaging is critical for good transplant survival outcomes, which may be less about reducing tumor burden and more about selecting biology. In this regard, TACE responsiveness has been proposed as a more accurate predictor of survival after transplant than tumor size and number. ${ }^{91,92}$

Finally, the use of living donor liver transplantation (LDLT) has increased the supply of available organs among HCC patients. The first adult LDLT took place in 1994, sparking optimism for increased organ supply. ${ }^{93}$ However, LDLT has been associated with higher rates of local recurrence, which may be attributable to several factors. First, living donations are often provided under urgent circumstances, 
and recipients frequently have more severe tumor burden at transplant. ${ }^{94}$ Second, fast-tracking to surgery with living donation may preclude selection for good biology by virtue of withstanding a waiting period..$^{95}$ Third, it is speculated that rapid organ regeneration is associated with growth factors and cytokines that may stimulate indolent HCC cells. ${ }^{96}$ Despite this potential drawback, multiple trials have since compared LDLT to brain dead (BDLT) or deceased donation (DDLT), with similar safety and long-term survival profiles. ${ }^{97}$

\section{Conclusion}

HCC incidence will continue to rise in the 21 st century, and unfortunately mortality remains high for the majority of patients. Multiple treatment options for HCC exist. Ablation, surgical resection, and transplantation are considered the only potentially curative therapies for candidate patients. Going forward, innovation in diagnostic and surveillance technology, improvements in locoregional treatment modalities, application of combinatorial therapy, and expanded indications for standard procedures hold promise for improving outcomes for HCC.

\section{Disclosure}

The authors report no conflicts of interest in this work.

\section{References}

1. Torre LA, Bray F, Siegel RL, Ferlay J, Lortet-Tieulent J, Jemal A. Global cancer statistics, 2012. CA Cancer J Clin. 2015;65(2):87-108.

2. Choo SP, Tan WL, Goh BK, Tai WM, Zhu AX. Comparison of hepatocellular carcinoma in Eastern versus Western populations. Cancer. Epub 2016 Sep 13.

3. El-Serag HB. Hepatocellular carcinoma. $N$ Engl J Med. 2011; 365(12):1118-1127.

4. El-Serag HB, Kanwal F. Epidemiology of hepatocellular carcinoma in the United States: where are we? Where do we go? Hepatology. 2014;60(5):1767-1775.

5. Song P, Tobe RG, Inagaki Y, et al. The management of hepatocellular carcinoma around the world: a comparison of guidelines from 2001 to 2011. Liver Int. 2012;32(7):1053-1063.

6. Trevisani F, De Notariis S, Rapaccini G, et al. Semiannual and annual surveillance of cirrhotic patients for hepatocellular carcinoma: effects on cancer stage and patient survival (Italian Experience). Am J Gastroenterol. 2002;97(3):734-744.

7. Santagostino E, Colombo M, Rivi M, et al. A 6-month versus a 12-month surveillance for hepatocellular carcinoma in 559 hemophiliacs infected with the hepatitis C virus. Blood. 2003;102(1):78-82.

8. Santi V, Trevisani F, Gramenzi A, et al. Semiannual surveillance is superior to annual surveillance for the detection of early hepatocellular carcinoma and patient survival. J Hepatol. 2010;53(2):291-297.

9. Trinchet JC, Chaffaut C, Bourcier V, et al. Ultrasonographic surveillance of hepatocellular carcinoma in cirrhosis: a randomized trial comparing 3- and 6-month periodicities. Hepatology. 2011;54(6):1987-1997.

10. Wang JH, Chang KC, Kee KM, et al. Hepatocellular carcinoma surveillance at 4- Vs. 12-month intervals for patients with chronic viral hepatitis: a randomized study in community. Am J Gastroenterol. 2013;108(3):416-424.
11. Kokudo N, Hasegawa K, Akahane M, et al. Evidence-based clinical practice guidelines for hepatocellular carcinoma: the Japan society of hepatology 2013 update (3rd Jsh-Hcc guidelines). Hepatol Res. 2015;45(2):123-127.

12. Cucchetti A, Garuti F, Pinna AD, Trevisani F; Italian Liver Cancer (ITA.LI.CA) Group. Length time bias in surveillance for hepatocellular carcinoma diagnosis and how to avoid it. Hepatol Res. 2016;46(12):1275-1280.

13. Kansagara D, Papak J, Pasha AS, et al. Screening for hepatocellular carcinoma in chronic liver disease: a systematic review. Ann Intern Med. 2014;161(4):261-269.

14. Lok AS, Sterling RK, Everhart JE, et al. Des-gamma-carboxy prothrombin and alpha-fetoprotein as biomarkers for the early detection of hepatocellular carcinoma. Gastroenterology. 2010;138(2):493-502.

15. Di Bisceglie AM, Sterling RK, Chung RT, et al. Serum alpha-fetoprotein levels in patients with advanced hepatitis $\mathrm{C}$ : results from the halt-C trial. J Hepatol. 2005;43(3):434-441.

16. Hu B, Tian X, Sun J, Meng X. Evaluation of individual and combined applications of serum biomarkers for diagnosis of hepatocellular carcinoma: a meta-analysis. Int J Mol Sci. 2013;14(12):23559-23580.

17. Gounder PP, Bulkow LR, Meltzer MI, et al. Cost-effectiveness analysis of hepatocellular carcinoma screening by combinations of ultrasound and alpha-fetoprotein among Alaska native people, 1983-2012. Int $J$ Circumpolar Health. 2016;75(1):31115.

18. Saab S, Ly D, Nieto J, et al. Hepatocellular carcinoma screening in patients waiting for liver transplantation: a decision analytic model. Liver Transpl. 2003;9(7):672-681.

19. Goldberg DS, Valderrama A, Kamalakar R, Sansgiry SS, Babajanyan S, Lewis JD. Hepatocellular carcinoma surveillance among cirrhotic patients with commercial health insurance. J Clin Gastroenterol. 2016;50(3):258-265.

20. Palmer LB, Kappelman MD, Sandler RS, Hayashi PH. Surveillance for hepatocellular carcinoma in a Medicaid cirrhotic population. $J$ Clin Gastroenterol. 2013;47(8):713-718.

21. Davila JA, Henderson L, Kramer JR, et al. Utilization of surveillance for hepatocellular carcinoma among hepatitis $\mathrm{C}$ virus-infected veterans in the United States. Ann Intern Med. 2011;154(2):85-93.

22. Goldberg DS, Taddei TH, Serper M, et al. Identifying barriers to hepatocellular carcinoma surveillance in a national sample of patients with cirrhosis. Hepatology. 2017;65(3):864-874.

23. Singal AG, Yopp A, Skinner SC, Packer M, Lee WM, Tiro JA. Utilization of hepatocellular carcinoma surveillance among American patients: a systematic review. J Gen Intern Med. 2012;27(7):861-867.

24. Hearn B, Chasan R, Bichoupan K, et al. Low adherence of HIV providers to practice guidelines for hepatocellular carcinoma screening in HIV/hepatitis B coinfection. Clin Infect Dis. 2015;61(11):1742-1748.

25. Joshi K, Mendler M, Gish R, et al. Hepatocellular carcinoma surveillance: a national survey of current practices in the USA. Dig Dis Sci. 2014;59(12):3073-3077.

26. Henrion J, Libon E, De Maeght S, et al. Surveillance for hepatocellular carcinoma: compliance and results according to the aetiology of cirrhosis in a cohort of 141 patients. Acta Gastroenterol Belg. 2000;63(1):5-9.

27. Singal AG, Volk ML, Rakoski MO, et al. Patient involvement in healthcare is associated with higher rates of surveillance for hepatocellular carcinoma. J Clin Gastroenterol. 2011;45(8):727-732.

28. Farvardin S, Patel J, Khambaty M, et al. Patient-reported barriers are associated with lower Hcc surveillance rates in patients with cirrhosis. Hepatology. 2017;65(3):875-884.

29. Gravitz L. Introduction: a smouldering public-health crisis. Nature. 2011;474(7350):S2-S4.

30. Alter MJ, Hadler SC, Judson FN, et al. Risk factors for acute non-A, non-B hepatitis in the United States and association with hepatitis C virus infection. JAMA. 1990;264(17):2231-2235.

31. Morgan TR, Ghany MG, Kim HY, et al. Outcome of sustained virological responders with histologically advanced chronic hepatitis C. Hepatology. 2010;52(3):833-844. 
32. Recommendations for prevention and control of hepatitis $\mathrm{C}$ virus (HCV) infection and HCV-related chronic disease. Centers for Disease Control and Prevention. MMWR Recomm Rep. 1998;47(RR-19):1-39.

33. Smith BD, Morgan RL, Beckett GA, Falck-Ytter Y, Holtzman D, Ward JW. Hepatitis C virus testing of persons born during 1945-1965: recommendations from the Centers for Disease Control and Prevention. Ann Intern Med. 2012;157(11):817-822.

34. Farinati F, Sergio A, Baldan A, et al. Early and very early hepatocellular carcinoma: when and how much do staging and choice of treatment really matter? A multi-center study. BMC Cancer. 2009;9:33.

35. Wang JH, Changchien $\mathrm{CS}, \mathrm{Hu} \mathrm{TH}$, et al. The efficacy of treatment schedules according to Barcelona clinic liver cancer staging for hepatocellular carcinoma - survival analysis of 3892 patients. Eur $J$ Cancer. 2008;44(7):1000-1006.

36. Bruix J, Sherman M; Practice Guidelines Committee AAftSoLD. Management of hepatocellular carcinoma. Hepatology. 2005;42(5):1208-1236.

37. Sherman M. Alphafetoprotein: an obituary. J Hepatol. 2001; 34(4):603-605.

38. Benson AB 3rd, Abrams TA, Ben-Josef E, et al. NCCN clinical practice guidelines in oncology: hepatobiliary cancers. J Natl Compr Canc Netw. 2009;7(4):350-391.

39. Singal A, Volk ML, Waljee A, et al. Meta-analysis: surveillance with ultrasound for early-stage hepatocellular carcinoma in patients with cirrhosis. Aliment Pharmacol Ther. 2009;30(1):37-47.

40. Cucchetti A, Vivarelli M, Piscaglia F, et al. Tumor doubling time predicts recurrence after surgery and describes the histological pattern of hepatocellular carcinoma on cirrhosis. J Hepatol. 2005;43(2):310-316.

41. Sherman M. Pathogenesis and screening for hepatocellular carcinoma. Clin Liver Dis. 2004;8(2):419-443,viii.

42. Yoon SH, Lee JM, So YH, et al. Multiphasic MDCT enhancement pattern of hepatocellular carcinoma smaller than $3 \mathrm{Cm}$ in diameter: tumor size and cellular differentiation. AJR Am J Roentgenol. 2009;193(6):W482-W489.

43. Park YN, Kojiro M, Di Tommaso L, et al. Ductular reaction is helpful in defining early stromal invasion, small hepatocellular carcinomas, and dysplastic nodules. Cancer. 2007;109(5):915-923.

44. Barone C, Koeberle D, Metselaar H, Parisi G, Sansonno D, Spinzi G. Multidisciplinary approach for Hcc patients: hepatology for the oncologists. Ann Oncol. 2013;24(suppl 2):ii15-ii23.

45. Bruix J, Gores GJ, Mazzaferro V. Hepatocellular carcinoma: clinical frontiers and perspectives. Gut. 2014;63(5):844-855.

46. Forner A, Reig ME, de Lope CR, Bruix J. Current strategy for staging and treatment: the BCLC update and future prospects. Semin Liver Dis. 2010;30(1):61-74.

47. Forner A, Llovet JM, Bruix J. Hepatocellular carcinoma. Lancet. 2012;379(9822):1245-1255.

48. Liu JH, Chen PW, Asch SM, Busuttil RW, Ko CY. Surgery for hepatocellular carcinoma: does it improve survival? Ann Surg Oncol. 2004;11(3):298-303.

49. Torzilli G, Belghiti J, Kokudo N, et al. A snapshot of the effective indications and results of surgery for hepatocellular carcinoma in tertiary referral centers: is it adherent to the EASL/AASLD Recommendations? An observational study of the HCC East-West Study Group. Ann Surg. 2013;257(5):929-937.

50. Zaydfudim VM, Vachharajani N, Klintmalm GB, et al. Liver resection and transplantation for patients with hepatocellular carcinoma beyond Milan criteria. Ann Surg. 2016;264(4):650-658.

51. Zhong JH, Wu FX, Li H. Hepatic resection associated with good survival for selected patients with multinodular hepatocellular carcinoma. Tumour Biol. 2014;35(9):8355-8358.

52. Hoekstra LT, de Graaf W, Nibourg GA, et al. Physiological and biochemical basis of clinical liver function tests: a review. Ann Surg. 2013;257(1):27-36.

53. Hayashi H, Beppu T, Okabe H, et al. Functional assessment versus conventional volumetric assessment in the prediction of operative outcomes after major hepatectomy. Surgery. 2015;157(1):20-26.
54. Kubota K, Makuuchi M, Kusaka K, et al. Measurement of liver volume and hepatic functional reserve as a guide to decision-making in resectional surgery for hepatic tumors. Hepatology. 1997;26(5):1176-1181.

55. Poon RT, Fan ST, Lo CM, et al. Extended hepatic resection for hepatocellular carcinoma in patients with cirrhosis: is it justified? Ann Surg. 2002;236(5):602-611.

56. Farges O, Belghiti J, Kianmanesh R, et al. Portal vein embolization before right hepatectomy: prospective clinical trial. Ann Surg. 2003;237(2):208-217.

57. May BJ, Madoff DC. Portal vein embolization: rationale, technique, and current application. Semin Intervent Radiol. 2012;29(2):81-89.

58. Seo DD, Lee HC, Jang MK, et al. Preoperative portal vein embolization and surgical resection in patients with hepatocellular carcinoma and small future liver remnant volume: comparison with transarterial chemoembolization. Ann Surg Oncol. 2007;14(12):3501-3509.

59. Choti MA, Geschwind JF. Preoperative sequential TACE and PVE to increase resectability in the cirrhotic patient with Hcc. Gastrointest Cancer Res. 2008;2(1):47-48.

60. Cucchetti A, Ercolani G, Vivarelli M, et al. Is portal hypertension a contraindication to hepatic resection? Ann Surg. 2009;250(6):922-928.

61. Ishizawa T, Hasegawa K, Aoki T, et al. Neither multiple tumors nor portal hypertension are surgical contraindications for hepatocellular carcinoma. Gastroenterology. 2008;134(7):1908-1916.

62. Berzigotti A, Reig M, Abraldes JG, Bosch J, Bruix J. Portal hypertension and the outcome of surgery for hepatocellular carcinoma in compensated cirrhosis: a systematic review and meta-analysis. Hepatology. 2015;61(2):526-536.

63. Nguyen KT, Gamblin TC, Geller DA. World review of laparoscopic liver resection-2,804 patients. Ann Surg. 2009;250(5):831-841.

64. Cheung TT, Poon RT, Yuen WK, et al. Long-term survival analysis of pure laparoscopic versus open hepatectomy for hepatocellular carcinoma in patients with cirrhosis: a single-center experience. Ann Surg. 2013;257(3):506-511.

65. Chen J, Li H, Liu F, Li B, Wei Y. Surgical outcomes of laparoscopic versus open liver resection for hepatocellular carcinoma for various resection extent. Medicine (Baltimore). 2017;96(12):e6460.

66. Shimada M, Hashizume M, Maehara S, et al. Laparoscopic hepatectomy for hepatocellular carcinoma. Surg Endosc. 2001;15(6): 541-544.

67. Gruttadauria S, Tropea A, Pagano D, et al. Mini-invasive approach contributes to expand the indication for liver resection for hepatocellular carcinoma without increasing the incidence of posthepatectomy liver failure and other perioperative complications: a single-center analysis. J Laparoendosc Adv Surg Tech A. 2016;26(6):439-446.

68. Morise Z, Ciria R, Cherqui D, Chen KH, Belli G, Wakabayashi G. Can we expand the indications for laparoscopic liver resection? A systematic review and meta-analysis of laparoscopic liver resection for patients with hepatocellular carcinoma and chronic liver disease. J Hepatobiliary Pancreat Sci. 2015;22(5):342-352.

69. Brown KM, Geller DA. What is the learning curve for laparoscopic major hepatectomy? J Gastrointest Surg. 2016;20(5):1065-1071.

70. Xu Q, Kobayashi S, Ye X, Meng X. Comparison of hepatic resection and radiofrequency ablation for small hepatocellular carcinoma: a meta-analysis of 16,103 patients. Sci Rep. 2014;4:7252.

71. Huang J, Yan L, Cheng Z, et al. A randomized trial comparing radiofrequency ablation and surgical resection for Hcc conforming to the Milan criteria. Ann Surg. 2010;252(6):903-912.

72. Lu MD, Kuang M, Liang LJ, et al. [Surgical resection versus percutaneous thermal ablation for early-stage hepatocellular carcinoma: a randomized clinical trial]. Zhonghua Yi Xue Za Zhi. 2006;86:801-805.

73. Feng K, Yan J, Li X, et al. A randomized controlled trial of radiofrequency ablation and surgical resection in the treatment of small hepatocellular carcinoma. J Hepatol. 2012;57(4):794-802.

74. Kang TW, Rhim H, Lee J, et al. Magnetic resonance imaging with gadoxetic acid for local tumour progression after radiofrequency ablation in patients with hepatocellular carcinoma. Eur Radiol. 2016; 26(10):3437-3446. 
75. Kim HD, Lim YS, Han S, et al. Evaluation of early-stage hepatocellular carcinoma by magnetic resonance imaging with gadoxetic acid detects additional lesions and increases overall survival. Gastroenterology. 2015;148(7):1371-1382.

76. Ahn SS, Kim MJ, Lim JS, Hong HS, Chung YE, Choi JY. Added value of gadoxetic acid-enhanced hepatobiliary phase MR imaging in the diagnosis of hepatocellular carcinoma. Radiology. 2010;255(2):459-466.

77. Koda M, Tokunaga S, Okamoto T, et al. Clinical usefulness of the ablative margin assessed by magnetic resonance imaging with GdEOB-DTPA for radiofrequency ablation of hepatocellular carcinoma. J Hepatol. 2015;63(6):1360-1367.

78. Liu PH, Hsu CY, Hsia CY, et al. Surgical resection versus radiofrequency ablation for single hepatocellular carcinoma $\leq 2 \mathrm{~cm}$ in a propensity score model. Ann Surg. 2016;263(3):538-545.

79. Rahbari NN, Mehrabi A, Mollberg NM, et al. Hepatocellular carcinoma: current management and perspectives for the future. Ann Surg. 2011;253(3):453-469.

80. Koniaris LG, Levi DM, Pedroso FE, et al. Is surgical resection superior to transplantation in the treatment of hepatocellular carcinoma? Ann Surg. 2011;254(3):527-537. discussion 537-538.

81. Parikh ND, Hutton D, Marrero W, Sanghani K, Xu Y, Lavieri M. Projections in donor organs available for liver transplantation in the United States: 2014-2025. Liver Transpl. 2015;21(6):855-863.

82. Ioannou GN, Perkins JD, Carithers RL Jr. Liver transplantation for hepatocellular carcinoma: impact of the meld allocation system and predictors of survival. Gastroenterology. 2008;134(5):1342-1351.

83. Roayaie K, Feng S. Allocation policy for hepatocellular carcinoma in the MELD era: room for improvement? Liver Transpl. 2007; 13(11 suppl 2):S36-S43.

84. Prasad MA, Kulik LM. The role of bridge therapy prior to orthotopic liver transplantation. JNatl Compr Canc Netw. 2014;12(8):1183-1190. quiz 1191.

85. Iwatsuki S, Gordon RD, Shaw BW Jr, Starzl TE. Role of liver transplantation in cancer therapy. Ann Surg. 1985;202(4):401-407.

86. Mazzaferro V, Regalia E, Doci R, et al. Liver transplantation for the treatment of small hepatocellular carcinomas in patients with cirrhosis. N Engl J Med. 1996;334(11):693-699.

87. Yao FY, Xiao L, Bass NM, Kerlan R, Ascher NL, Roberts JP. Liver transplantation for hepatocellular carcinoma: validation of the Ucsfexpanded criteria based on preoperative imaging. Am J Transplant. 2007;7(11):2587-2596.

88. Mazzaferro V, Llovet JM, Miceli R, et al. Predicting survival after liver transplantation in patients with hepatocellular carcinoma beyond the Milan criteria: a retrospective, exploratory analysis. Lancet Oncol. 2009;10(1):35-43.

89. Duffy JP, Vardanian A, Benjamin E, et al. Liver transplantation criteria for hepatocellular carcinoma should be expanded: a 22-year experience with 467 patients at UCLA. Ann Surg. 2007;246(3):502-509. discussion 509-511.

90. Chapman WC, Majella Doyle MB, Stuart JE, et al. Outcomes of neoadjuvant transarterial chemoembolization to downstage hepatocellular carcinoma before liver transplantation. Ann Surg. 2008;248(4):617-625.

91. Otto G, Schuchmann M, Hoppe-Lotichius M, et al. How to decide about liver transplantation in patients with hepatocellular carcinoma: size and number of lesions or response to TACE? J Hepatol. 2013;59(2):279-284.
92. Vitale A, D'Amico F, Frigo AC, et al. Response to therapy as a criterion for awarding priority to patients with hepatocellular carcinoma awaiting liver transplantation. Ann Surg Oncol. 2010;17(9):2290-2302.

93. Dutkowski P, De Rougemont O, Mullhaupt B, Clavien PA. Current and future trends in liver transplantation in Europe. Gastroenterology. 2010;138(3): 802-809.e1-e4.

94. Fisher RA, Kulik LM, Freise CE, et al. Hepatocellular carcinoma recurrence and death following living and deceased donor liver transplantation. Am J Transplant. 2007;7(6):1601-1608.

95. Kulik L, Abecassis M. Living donor liver transplantation for hepatocellular carcinoma. Gastroenterology. 2004;127:S277-S282.

96. Park MS, Lee KW, Suh SW, et al. Living-donor liver transplantation associated with higher incidence of hepatocellular carcinoma recurrence than deceased-donor liver transplantation. Transplantation. 2014;97(1):71-77.

97. Azoulay D, Audureau E, Bhangui P, et al. Living or brain-dead donor liver transplantation for hepatocellular carcinoma: a multicenter, western, Intent-to-Treat Cohort Study. Ann Surg. Epub 2016 Sep 9.

98. Heimbach J, Kulik LM, Finn R, et al. AASLD guidelines for the treatment of hepatocellular carcinoma. Hepatology. Epub 2017 Jan 28.

99. European Association for the Study of the Liver; European Organisation for Research and Treatment of Cancer. EASL-EORTC clinical practice guidelines: management of hepatocellular carcinoma. J Hepatol. 2012;56(4):908-943.

100. Kudo M, Matsui O, Izumi N, et al. JSH consensus-based clinical practice guidelines for the management of hepatocellular carcinoma: 2014 update by the Liver Cancer Study Group of Japan. Liver Cancer. 2014;3(3-4):458-468

101. Omata M, Lesmana LA, Tateishi R, et al. Asian Pacific Association for the study of the liver consensus recommendations on hepatocellular carcinoma. Hepatol Int. 2010;4(2):439-474.

102. Azab M, Zaki S, El-Shetey AG, et al. Radiofrequency ablation combined with percutaneous ethanol injection in patients with hepatocellular carcinoma. Arab J Gastroenterol. 2011;12(3):113-118.

103. Peng ZW, Zhang YJ, Chen MS, et al. Radiofrequency ablation with or without transcatheter arterial chemoembolization in the treatment of hepatocellular carcinoma: a prospective randomized trial. J Clin Oncol. 2013;31(4):426-432.

104. Bian H, Zheng JS, Nan G, et al. Randomized trial of [131i] metuximab in treatment of hepatocellular carcinoma after percutaneous radiofrequency ablation. J Natl Cancer Inst. 2014;106(9):dju239.

105. Chen K, Chen G, Wang H, et al. Increased survival in hepatocellular carcinoma with iodine-125 implantation plus radiofrequency ablation: a prospective randomized controlled trial. J Hepatol. 2014;61(6):1304-1311.

106. Liu H, Wang ZG, Fu SY, et al. Randomized clinical trial of chemoembolization plus radiofrequency ablation versus partial hepatectomy for hepatocellular carcinoma within the Milan criteria. Br J Surg. 2016;103(4):348-356

107. Thrift AP, El-Serag HB, Kanwal F. Global epidemiology and burden of Hcv infection and Hcv-related disease. Nat Rev Gastroenterol Hepatol. 2017;14(2):122-132.

108. Petrick JL, Kelly SP, Altekruse SF, McGlynn KA, Rosenberg PS. Future of hepatocellular carcinoma incidence in the United States forecast through 2030. J Clin Oncol. 2016;34(15):1787-1794.
Journal of Hepatocellular Carcinoma

\section{Publish your work in this journal}

The Journal of Hepatocellular Carcinoma is an international, peerreviewed, open access journal that offers a platform for the dissemination and study of clinical, translational and basic research findings in this rapidly developing field. Development in areas including, but not limited to, epidemiology, vaccination, hepatitis therapy, pathology and

\section{Dovepress}

molecular tumor classification and prognostication are all considered for publication. The manuscript management system is completely online and includes a very quick and fair peer-review system, which is all easy to use. Visit http://www.dovepress.com/testimonials.php to read real quotes from published authors. 